\title{
Improved Method for Detecting Differentially Expressed Genes Using cDNA Indexing
}

BioTechniques 28:958-964 (May 2000)

C.J. Shaw-Smith 1,2 , A.J.

Coffey $^{2}$, E. Huckle ${ }^{2}$, J.

Durham $^{2}$, E.A. Campbell 2 , T.C. Freeman ${ }^{2}$, J.R.F. Walters ${ }^{1}$ and D.R. Bentley ${ }^{2}$

${ }^{1}$ Hammersmith Hospital, London, and ${ }^{2}$ The Sanger

Centre, Cambs, UK

\begin{abstract}
In cDNA indexing, differentially expressed genes are identified by the display of specific, corresponding subsets of cDNA. Subdivision of the cDNA population is achieved by the sequence-specific ligation of adapters to the overhangs created by class IIS restriction enzymes. However, inadequate specificity of ligation leads to redundancy between different adapter subsets. We evaluate the incidence of mismatches between adapters and class IIS restriction fragments during ligation and describe a modified set of conditions that improves ligation specificity. The improved protocol reduces redundancy between amplified cDNA subsets, which leads to a lower number of bands per lane of the differential display gel, and therefore simplifies analysis. We confirm the validity of this revised protocol by identifying five differentially expressed genes in mouse duodenum and ileum.
\end{abstract}

\section{INTRODUCTION}

Systematic differential display methods (10), such as cDNA indexing $(6,8)$, offer a potential solution to some of the technical difficulties encountered in classical differential display (7). These include a high false positive rate, lack of $3^{\prime}$ end representation and redundancy between cDNA subsets. Although these problems have to some extent been mitigated by recent improvements (9), there continues to be interest in alternative approaches.

In one of these approaches, cDNA indexing $(6,8)$, specific subsets of cDNA fragments are generated by restriction digestion and then isolated by ligating an adapter to the resulting cohesive ends before amplification by PCR and gel display. The initial subdivision of the cDNA population is achieved by using either different restriction enzymes $(5,11)$ or the different $5^{\prime}$ overhangs created by a class IIS restriction enzyme $(6,8)$. Redundancy between cDNA subsets has proved to be a problem, however. In the methods that employ conventional restriction enzymes, overlap between subsets created by different enzymes is inevitable. When a class IIS enzyme is used, smaller cDNA subsets are created, but redundancy attributed to a lack of specificity of DNA ligase has been observed (6). We have therefore evaluated in detail the specificity with which the adapters are ligated to cohesive ends of class IIS restriction fragments.

Here, we describe an improved protocol for cDNA indexing of Kato (6), which is based on enhanced ligation specificity. We demonstrate its effectiveness by identifying differentially expressed genes in mouse small intestine.

\section{MATERIALS AND METHODS}

\section{Preparation of cDNA}

Duodenal and ileal tissues were isolated from six- to eight-week-old C57BL6 mice and snap-frozen. Total RNA was extracted using the Invisorb ${ }^{\mathrm{TM}}$ Total RNA extraction kit (Bioline, London, UK). Double-stranded cDNA was synthesized from $50 \mu \mathrm{g}$ total RNA using the cDNA synthesis system (Life Technologies, Paisley, UK). The reverse transcription primer was 5'-CAGGAAACAGCTATGACCT $_{17}$ (AGC)(AGCT)3 '. One microgram of the cDNA was digested with $5 \mathrm{U}$ FokI or BsmAI (New England Biolabs, Beverly, MA, USA) in a $50-\mu \mathrm{L}$ reaction volume.

\section{Ligation of Adapter}

Oligonucleotides for vectorette adapters were HPLC-purified (Oswel, Southampton, UK). Each adapter contains a 53-bp constant oligonucleotide (BPBII: 5'-CTCTCCCTTCTCGAATCGTAACCGTTCGTACGAGAATCGCTGTCCTCTCCTTG-3') and a 57-bp variable oligonucleotide (BPBIA: 5'GATCCAAGGAGAGGACGCTGTCTGTCGAAGGTAAGGAACGGACGAGAGAAGGGAGAG-3', with the overhang 5'-GATC). Oligonucleotides with the overhangs $5^{\prime}$-GATG, 5'-GATA, 5'-GATT, 5'-GAGC, 5'-GACC, 5'GAAC, 5'-GGTC, 5'-GCTC, 5'-GTTC, 
5'-CATC, 5'-TATC, 5'-AATC and 5'GCGC were also synthesized. The oligonucleotides were annealed in 25 $\mathrm{mM} \mathrm{NaCl}$ at $65^{\circ} \mathrm{C}$ for $15 \mathrm{~min}$ and then allowed to cool to room temperature to form the adapter. Digested cDNA (200 ng) was ligated to $17,1.7$ or $0.17 \mathrm{pmol}$ adapter in a $10-\mu \mathrm{L}$ reaction volume with 40 U Taq DNA ligase (New England Biolabs) or 1 U T4 DNA ligase (Life Technologies) at different temperatures and for different durations (see Results). On completion, the mixture was diluted by adding $40 \mu \mathrm{L} 10 \mathrm{mM}$ Tris- $\mathrm{HCl}(\mathrm{pH}$ 8.0), 0.1 mM EDTA.

\section{PCR Amplification and Electrophoresis}

The primary PCR was performed with $5 \mu \mathrm{L}$ ligation reaction in a final volume of $15 \mu \mathrm{L}$ using 224 (5'CGAATCGTAACCGTTCGTACGAGAATCGCT-3') and M13R (5'-CAGGAAACAGCTATGACC- $3^{\prime}$ ) on a PTC$225^{\mathrm{TM}}$ thermal cycler (MJ Research, Waltham, MA, USA). PCR cycling conditions were $94^{\circ} \mathrm{C}$ for $5 \mathrm{~min} ; 35 \mathrm{cy}$ cles of $93^{\circ} \mathrm{C}$ for $30 \mathrm{~s}, 60^{\circ} \mathrm{C}$ for $30 \mathrm{~s}$ and $72^{\circ} \mathrm{C}$ for $30 \mathrm{~s}$; and finally $72^{\circ} \mathrm{C}$ for 5 min. The reaction mixture contained 6.7 mM Mg ${ }^{++}$buffer, 0.9 U Taq DNA polymerase (PE Biosystems, Foster City, CA, USA), dNTPs at a concentration of $0.5 \mathrm{mM} / \mu \mathrm{L}$ and primers at a concentration of $1.1 \mu \mathrm{M}(\mathrm{M} 13 \mathrm{R})$ and $0.67 \mu \mathrm{M}$ (224). Two microliters of a 60 -fold dilution of the primary reaction were used in the secondary reaction, using M13R and 224 primers that were $5^{\prime}$ end-labeled with $\gamma^{32} \mathrm{P}$ dATP as described previously (3). Reaction constituents and cycling conditions were the same as for the primary reaction except that primer concentrations were $0.1 \mu \mathrm{M}$ (M13R) and $0.06 \mu \mathrm{M}(224)$, and 10 cycles of PCR were used. PCR products were separated by electrophoresis on a $4 \%$ denaturing polyacrylamide gel. Markers were a SauIIIA digestion of $\lambda$ phage. Gels were fixed, dried and analyzed by autoradiography.

\section{Selection and Sequencing of Bands for Further Analysis}

The gel was rehydrated by immersion in water for $20 \mathrm{~min}$. Bands chosen for further analysis were selected from the gel using a 20 -gauge needle and inoculated directly into the PCR mixture for reamplification. PCR (35 cycles) was performed using 224 and M13R, with the same cycling conditions as for the primary reaction. Products were sequenced directly (12). Sequences were analyzed using the BLASTN algorithm, and sequence data was displayed graphically using A. C. elegans database (4). Primers were designed to the sequences of bands picked for further investigation. PCR was performed on mouse duodenal and ileal cDNA using the same cycling conditions as the primary reaction. Northern analyses were performed in accordance with standard procedures (13). PCR products were labeled and hybridized as described (1).

\section{Subcloning of PCR Products}

Approximately $10 \mathrm{ng}$ primary PCR product were purified using Geneclean $^{\mathrm{TM}}$ II (Biosis 101, Vista, CA, USA) and ligated to $10 \mathrm{ng}$ pGEM $^{\circledR}$-T vector (Promega, Madison, WI, USA) according to the manufacturer's instructions. Inserts were amplified by PCR and sequenced.

\section{RESULTS}

The approach taken is similar to that described by Kato (6). Double-stranded cDNA was synthesized and digested with a class IIS restriction enzyme (FokI or BsmAI). Vectorette adapters with overhangs compatible with one of the 256 possible $5^{\prime}$ cohesive termini were ligated to aliquots of the digested cDNA. Following PCR amplification, the products were radiolabeled in a second round of PCR using end-labeled primers and displayed by high-resolution polyacrylamide gel electrophoresis. Bands that appeared to represent differentially expressed genes (on the basis of their relative intensities after amplification from duodenal and ileal samples) were purified for sequencing and further analysis.

In an initial evaluation of ligation specificity using T4 DNA ligase, mouse ileal cDNA digested with FokI was ligated to an adapter containing the unique 5 ' overhang GATC. Products were amplified by PCR, subcloned and 
sequenced. The specificity of the ligation reaction was judged by the number of occurrences of the correct matching of each base in the cDNA $5^{\prime}$ overhang with the corresponding base in the adapter. A high incidence of noncomplementary base pairings in all four base positions was found (data not shown).

To improve the specificity of the ligation reaction, we tested a series of different ligation reaction conditions using the same combination of adapter and cDNA. Ligation products were amplified, subcloned and sequenced as previously described. The initial ligation conditions tested were repeated for comparison (Figure 1, A). Of the modified reaction conditions, the best overall results (Table 1) (Figure 1, B4) were obtained using Taq DNA ligase instead of T4 DNA ligase, a 60-min reaction duration and a molar ratio of adapter:cDNA of 10:1. Under these conditions, most clones sequenced represented exactly matching ligation events, and very few $(2 / 60)$ (Table 1$)$ were amplified by mispriming of M13R or 224 from close sequence matches in the cDNAs. Greater specificity of ligation $(100 \%)$ was observed when a shorter reaction time (2 min) and a lower adapter:cDNA molar ratio $(1: 1)$ were used (Figure 1, B3). However, under these conditions, the proportion of sequences representing ligation events was lower [Table 1,7/24 (B3) versus 58/60 (B4)], and the proportion caused by mispriming was correspondingly higher.

The optimized conditions were tested more extensively using BsmAI-digested mouse ileal cDNA and 13 adapters with unique $5^{\prime}$ overhangs. The combined results of 139 ligations are shown in Figure 1, C. A high degree of specificity (over $90 \%$ ) between adapter and cDNA was seen at base positions 1 , 2 and 4 . Less specificity (56\%) was observed at base position 3 in the cDNA $5^{\prime}$ overhang. The frequencies of the eight possible mismatches that occurred in this base position are shown in Table 2. One wobble base pairing, GA, occurred most frequently in this position and accounted for 39/61 (64\%) of the mismatches. Of the clones that were amplified by mispriming of M13R or 224 in this experiment (80/219) (Table 1), 52/80 were found in sequences from analysis of three

Table 1. Number of Individual Clones Sequenced for Each Ligation Reaction Condition (A, B1-B4 and C; See Figure 2)

\begin{tabular}{|cccc|}
\hline Experiment & $\begin{array}{c}\text { Total No. of Clones } \\
\text { Sequenced }\end{array}$ & Indexed & Misprimed \\
\hline A & 19 & 19 & 0 \\
B1 & 31 & 5 & 26 \\
B2 & 34 & 34 & 0 \\
B3 & 24 & 7 & 17 \\
B4 & 60 & 58 & 2 \\
C & 219 & 139 & 80 \\
The total number is divided into two groups: an indexed group in which 3' cDNA & \\
restriction fragments were ligated to the adapter before amplification; and a mis- \\
primed group in which sequences were amplified by direct priming of 224 or \\
M13R from close matches in cDNA coding sequences. \\
\hline
\end{tabular}

adapters with the $5^{\prime}$ overhang sequences GATA, GATT and TATC. These sequences have a high $\mathrm{A} / \mathrm{T}$ content, and it is possible that they are less efficiently ligated, resulting in mispriming events that comprise a higher proportion of the total number of sequences obtained. When the same
cDNA fragment was found in two or more adapter subgroups, this result could be accounted for either by the wobble in base position 3 of the overhang or by the fact that the product was caused by mispriming of 224 or M13R on the cDNA, a ligation-independent process. Apart from this, there was

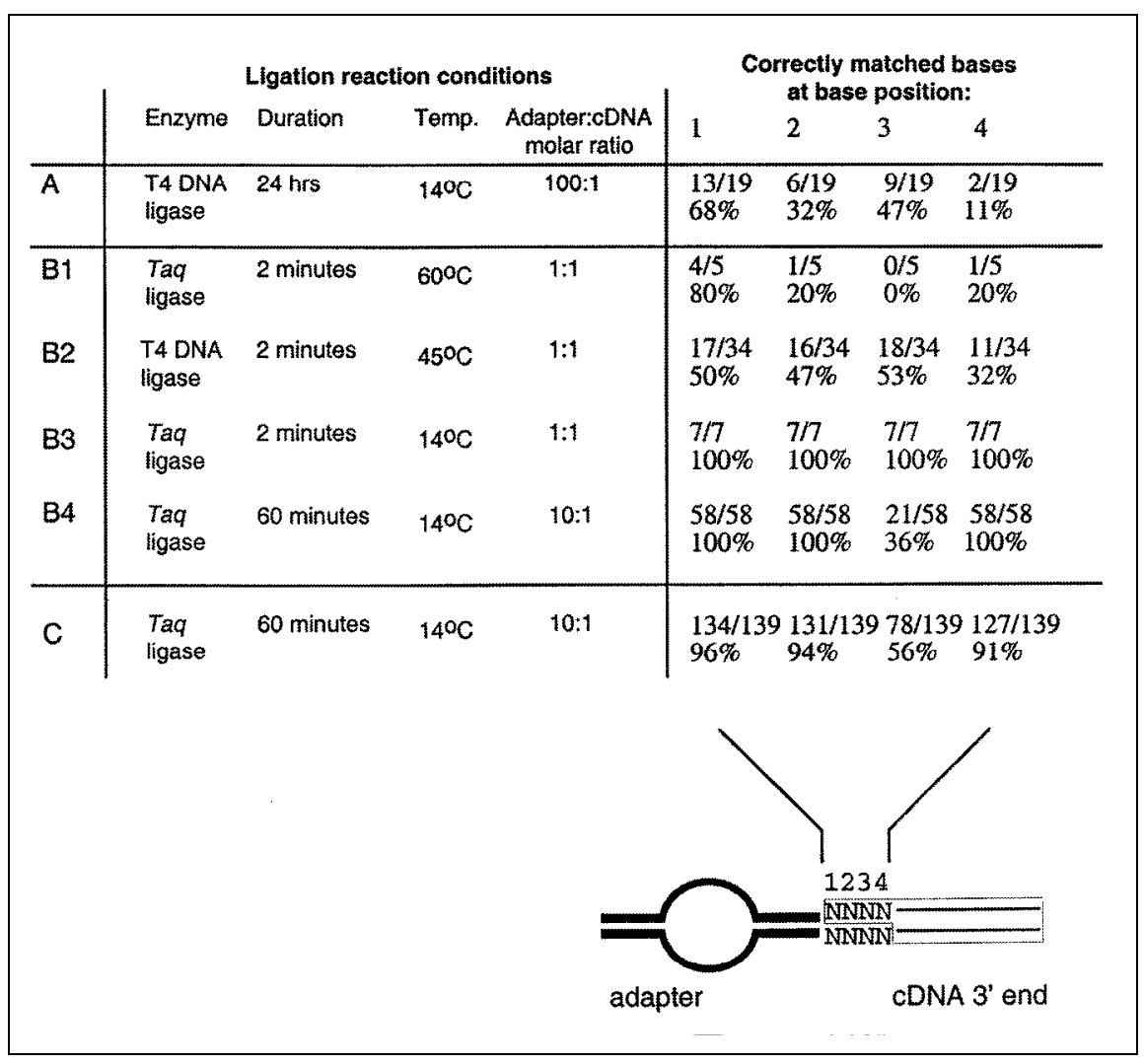

Figure 1. Data on ligation reaction specificity from sequencing clones obtained by cDNA indexing. The numbers and percentages of correctly matched base pairings in each base position (marked 1-4) of the cDNA $5^{\prime}$ overhang are shown for products obtained using different ligation reaction conditions, which are detailed to the left of the figure. A and B (1-4): GATC adapter; C pooled results for 13 different adapters. 
Table 2. Mismatches Occurring at Base Position 3 (See Figure 1) During Ligation of Adapters to BsmAI-Digested cDNA Population

\begin{tabular}{|c|c|c|c|c|c|c|c|c|}
\hline Mismatch & G-G & G-A & G-T & C-C & C-A & C-T & $\mathrm{T}-\mathrm{T}$ & A-A \\
\hline No. of occurrences & 1 & 39 & 12 & 0 & 3 & 0 & 0 & 6 \\
\hline
\end{tabular}

Table 3. Summary of Evaluation by RT-PCR and Northern Hybridization of Bands Selected from cDNA Indexing Gels

\begin{tabular}{|c|c|c|c|c|c|}
\hline \multirow[b]{2}{*}{ Band name } & \multirow{2}{*}{$\begin{array}{l}\text { Match to } \\
\text { EMBL Ac. } \\
\text { no. }\end{array}$} & \multirow[b]{2}{*}{ Gene name } & \multicolumn{2}{|c|}{ Expression } & \multirow{2}{*}{$\begin{array}{l}\text { Differential } \\
\text { expression } \\
\text { confirmed }\end{array}$} \\
\hline & & & RT-PCR & Northern & \\
\hline A6l1 & aa688697 & EST & 1 & 1 & yes \\
\hline A1D1 & u31966 & $\begin{array}{l}\text { mouse } \\
\text { carbonyl } \\
\text { reductase }\end{array}$ & $D>1$ & $\mathrm{D}$ & yes \\
\hline A9D1 & as A1D1 & & & & \\
\hline A9l1 & aa600608 & EST & $\mathrm{I}>\mathrm{D}$ & $\mathrm{I}>\mathrm{D}$ & yes \\
\hline A912 & aa997974 & EST & NDE & $1>D$ & Northern only \\
\hline B7I1 & ai070183 & EST & $1>D$ & $1>D$ & yes \\
\hline
\end{tabular}


minimal redundancy of sequence data between the different adapter subsets.

The ability of cDNA indexing to detect differentially expressed genes was investigated by comparing gene expression in mouse duodenum and ileum. Mouse duodenal and ileal dscDNA samples were digested with BsmAI and ligated in separate reactions to each of four different adapters. The number of bands seen per lane of the gel ranges between 15 and 25 . Six bands appeared to be differentially expressed based on differences in their relative intensities between the two tissues (Figure 2). Following gel purification and reamplification, these bands were sequenced directly without a prior cloning step. Two sequences, A1D1 and A9D1, were found to be identical. The sequence of the restriction fragment overhang was found to be 5'-GAGC in both cases, indicating that a mismatched ligation to the 5'-GATC adapter occurred, with a G-A mismatch at position 3-the most commonly identified mismatch. One sequence, A9I2, appeared to be the result of a mispriming event. The other four bands appeared to be from ligation events, based on the presence of a $B s m A I$ restriction site and an overhang sequence appropriate to the adapter.

Sequences obtained were used to search the European Molecular Biology Laboratory database using the BLAST algorithm (Table 3). One sequence (A1D1/A9D1) matched a previously identified mouse carbonyl reductase gene (14). A second sequence, A9I1, matched a mouse expressed sequence tag (aa600608.em_est1) that was found to be part of a Unigene cluster showing significant homology to human phosphomevalonate kinase gene (EMBL accession no. L77213) (2). The remaining three sequences matched mouse expressed sequence tags (Table 3). Differential expression was confirmed by Northern hybridization of duodenal and ileal total RNA for all five bands (Figure 3 and Table 3). For four of the five bands, differential expression was also confirmed by RT-PCR, but for the remaining one (A9I2), no difference in signal could be detected. For A1D1, our data are in agreement with a finding of stronger expression of a human carbonyl reductase gene in duodenum compared with ileum (15).

\section{DISCUSSION}

By improving ligation specificity, we show reproducible recovery of highly specific subsets of cDNA sequences by cDNA indexing, and we confirm the identification of five cDNAs that are differentially expressed in mouse duodenum and ileum. We extend the observation of Kato (6) that the ligation of unique adapters to class IIS restriction fragments may result in mismatched base pairing by a systematic analysis of cloned cDNA indexing products obtained under a variety of different ligation conditions. Our data show that the long ligation duration and high adapter:cDNA molar ratio used in Kato's protocol reduce the specificity of ligation. It is also apparent from our data that greater ligation specificity is ob- tained at lower rather than higher temperatures. Using the optimized conditions described, a higher degree $(>90 \%)$ of specificity was achieved at three of the four positions in the $5^{\prime} \mathrm{cDNA}$ overhang for 13 different adapters. In the remaining position (position 3, Figure 1), a wobble was observed, though one mismatch (G-A) was favored, suggesting that some specificity can be achieved and redundancy is not complete. The wobble position found does not correspond with that suggested by Kato (6), which was predicted to be at base position 4 of the cDNA overhang.

The higher ligation specificity that we report allows the reproducible division of cDNA populations into small subsets, with minimal overlap between each subset, reducing the number of bands appearing in each lane of the gel.

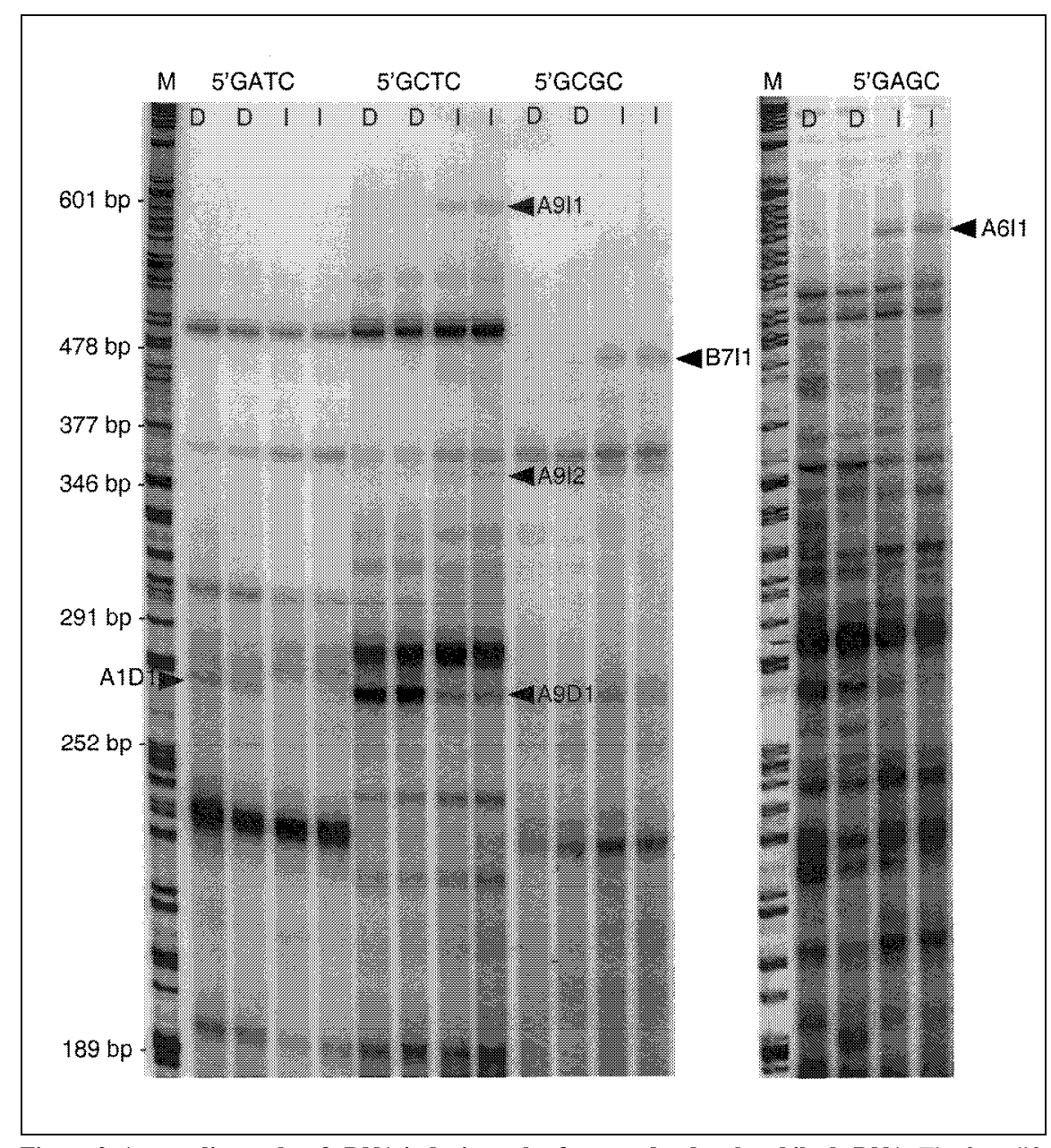

Figure 2. Autoradiographs of cDNA indexing gels of mouse duodenal and ileal cDNA. The four different adapters used are denoted above the lanes. The primary PCR was performed in duplicate as a check for reproducibility. Putative differentially expressed bands that were selected for sequencing are identified by arrowheads. $\mathrm{D}=$ duodenum, $\mathrm{I}=$ ileum; $\mathrm{M}=$ markers. 


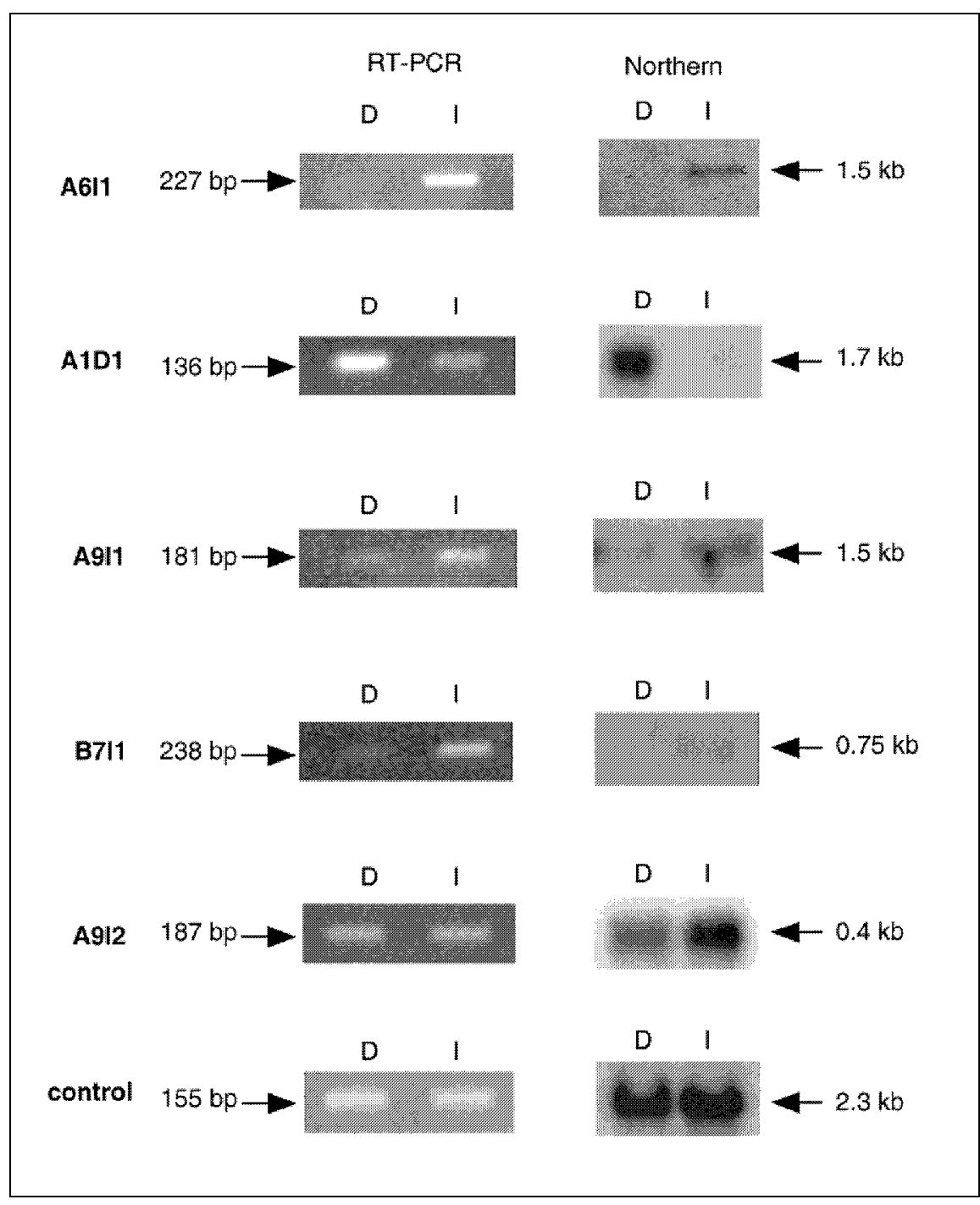

Figure 3. Confirmation of differential expression of isolated cDNAs by RT-PCR and Northern hybridization. The control is the mouse TIS11 gene (EMBL accession no. g54803). Sizes of PCR products and signals on Northern autoradiographs are indicated by arrows. $\mathrm{D}=$ duodenum; $\mathrm{I}=$ ileum.

Gel analysis and purification of products are thereby greatly facilitated because each band can be sequenced directly with no need for subcloning. This represents an advantage over previously reported differential display techniques in which cloning is often required before sequencing candidate bands.

\section{ACKNOWLEDGMENTS}

This work was funded by the Wellcome Trust. C.S.-S. was the recipient of a Wellcome Trust Clinical Training Fellowship. We would like to thank Robert Brooksbank for helpful discus- sions and David Vetrie for critical review of the manuscript.

\section{REFERENCES}

1.Bentley, D.R., C. Todd, J. Collins, J. Holland, I. Dunham, S. Hassock, A. Bankier and F. Gianelli. 1992. The development and application of automated gridding for efficient screening of yeast and bacterial ordered libraries. Genomics 12:534-541.

2.Chambliss, K.L., C.A. Slaughter, R. Schreiner, G.F. Hoffmann and K.M. Gibson. 1996. Molecular cloning of human phosphomevalonate kinase and identification of a consensus peroxisomal targeting sequence. J. Biol. Chem. 271:17330-17334.

3.Coffey, A.J., S.G. Gregory and C. Cole. 1996. Alu-PCR fingerprinting of YACs, p. 97-
114. In D. Markie (Ed.), YAC Protocols. Humana Press, Totowa, NJ.

4.Durbin, R. and J.T. Mieg. 1991. See http://www.sanger.ac.uk/Software/Acedb.

5.Ivanova, N.B. and A. V. Belyavsky. 1995. Identification of differentially expressed genes by restriction endonuclease-based gene expression fingerprinting. Nucleic Acids Res. 23:2954-2958.

6.Kato, K. 1995. Description of the entire mRNA population by a $3^{\prime}$ end cDNA fragment generated by class IIS restriction enzymes. Nucleic Acids Res. 23:3685-3690.

7.Liang, P., L. Averboukh, K. Keyomarsi, R. Sager and A.B. Pardee. 1992. Differential display and cloning of messenger RNAs from human breast cancer versus mammary epithelial cells. Cancer Res. 52:6966-6968.

8.Mahadeva, H., P. Starkey, F.N. Sheikh, C.R. Mundy and N.J. Samani. 1998. A simple and efficient method for the isolation of differentially expressed genes. J. Mol. Biol. 284:1391-1398.

9.Martin, K.J. and A.B. Pardee. 1999. Principles of differential display. Methods Enzymol. 303:234-258

10.Matz, M. and S. Lukyanov. 1998. Different strategies of differential display: areas of application. Nucleic Acids Res. 26:5537-5543.

11.Prashar, Y. and S.M. Weissman. 1996. Analysis of differential gene expression by display of $3^{\prime}$ end restriction fragments of cDNAs. Proc. Natl. Acad. Sci. USA 93:659663.

12.Prober, J.M., G.L. Trainor, R.J. Dam, F.W. Hobbs, C.W. Robertson, R.J. Zagursky, A.J. Cocuzza, M.A. Jensen and K. Baumeister. 1987. A system for rapid DNA sequencing with fluorescent chain-terminating dideoxynucleotides. Science 238:336-341.

13.Sambrook, J., T. Maniatis and E.F. Fritsch. 1989. Molecular Cloning: A Laboratory Manual. CSH Laboratory Press, Cold Spring Harbor, NY.

14.Wei, J., S.R. Dlouhy, A. Hara, B. Ghetti and M.E. Hodes. 1996. Cloning a cDNA for carbonyl reductase (Cbr) from mouse cerebellum: murine genes that express cbr map to chromosomes 16 and 11. Genomics 34:147148.

15.Wirth, P. and B. Wermuth. 1992. Immunohistochemical localization of carbonyl reductase in human tissues. J. Histochem. Cytochem. 40:1857-1863.

Received 28 June 1999; accepted 22 December 1999.

\section{Address correspondence to:}

Dr. Julian R.F. Walters

Gastroenterology Section

Imperial College School of Medicine

Hammersmith Hospital

London, W12 ONN

England, UK

Internet: julian.walters@ic.ac.uk 